\title{
The influence of instructions on the adjustment of scales
}

\author{
GERT HAUBENSAK and PETER PETZOLD \\ Justus Liebig University of Giessen, Giessen, Germany
}

\begin{abstract}
In each of three experiments, one group of subjects first judged the eight largest and another group first judged the eight smallest of a graded series of 16 squares according to size. After four blocks of trials, both groups were shifted to the total series. The subjects changed their scales only slightly when no specific instructions were given at the point of shift (Experiment 1). They were able to maintain their old scales or construct new ones, if instructed to do so (Experiment 2). They were also able to do both things in parallel (Experiment 3). An additional finding was that responses to the "old" stimuli (those being part of the training series) were slower than responses to the "new" ones (those introduced after the shift), independently by of instructions. Furthermore, responses to the old stimuli were faster when these had to be rated on the new scale, and responses to the new stimuli were faster when these had to be rated on the old scale. The findings are interpreted as supporting a conditioning theory of judgment.
\end{abstract}

Numerous studies (e.g., Di Lollo, 1964; Di Lollo \& Casseday, 1965; Johnson, 1949; Tresselt, 1947; Ward, 1987; Ward \& Lockhead, 1970; Wedell, 1984) have shown that first experience in a series of stimuli affects the judgment of the second series. Tresselt, for example, found that a group of subjects who had first judged the 4 heaviest of 12 weights called a heavier weight medium than did a group of subjects who first judged the 4 lightest weights. The discrepancy between the two groups rapidly diminished but did not disappear during the entire experiment. Similarly, in experiments with tones, the last 200 loudness judgments on the next day were still affected by the previous days' loudness scales (Ward \& Lockhead, 1970). Ward found similar effects in other types of psychophysical tasks, such as absolute magnitude estimation, ratio magnitude estimation with a variable standard, and crossmodality matching. Ward concluded that a previous session's stimulus-response mappings can have dramatic, although not always consistent, effects on scaling responses during later sessions.

In none of his experiments, however, were subjects warned of a possible shift in the range of stimuli, nor were they told how to respond. In the experiments of Di Lollo (1964), Di Lollo and Casseday (1965), and Wedell (1984), the presentations proceeded smoothly from the training to the test series without any interruption or warning at the point of shift. Tresselt (1947) interrupted the presentations for $5 \mathrm{~min}$, Ward (1987) and Ward and Lockhead (1970) con-

This study was supported by the Deutsche Forschungsgemeinschaft (DFG) under Project No. Ha 936/6. The study was presented in part at the 17th Annual Meeting of the International Society for Psychophysics, Leipzig, October 2001. Correspondence should be addressed to G. Haubensak, Department of Psychology, Justus Liebig University, Otto-Behaghel-Str.10,35394Giessen, Germany (e-mail: gert.haubensak@ psychol.uni-giessen.de). tinued only on the next day, but the subjects were never told that they would be judging a shifted set of stimuli. Thus, it is not surprising that two thirds of Tresselt's subjects voiced their surprise when they were given first a weight well beyond the range of those in the practice series; a few stated spontaneously that the stimuli must belong to a new series.

After noticing the shift, some subjects might have decided to maintain their old scales, others might have tried to establish new ones, and still others might have resorted to a compromise by shifting their scales slightly. This would account for the large individual differences usually found in such experiments. Ward (1987), for example, noted that a few subjects adjusted themselves to a shift in loudness completely, whereas others kept to their old scales. Similarly, in an experiment with line spacings (Parducci, 1954), 5 of the 13 subjects did not shift their scales at all after an extension of the series beyond its upper end. In experiments with a reversal of stimulus frequencies (Wedell, 1984), two fifths of the subjects also showed little adjustment to the reversal, one third showed almost complete adjustment, and the remainder showed partial adjustment.

Self-reports suggest that subjects are able to control the amount of adjustment. In Wedell's (1984) experiment with a reversal of stimulus frequencies, one of the more articulate subjects reported the following:

At first the squares were quite large, and I set my scale to the first ten squares. After that a greater percentage of squares were smaller. After noticing this change I thought that I should reevaluate my ratings, but if I did reevaluate my scales, the early ratings of my early squares would be insignificant. Bottom line: I did slightly reevaluate the ratings of "large" to "small" squares.

(p. 135, italics by Wedell)

Reports such as these suggest that the adjustment of scales can be manipulated by giving subjects specific in- 
structions to modify or not to modify scales at the point of shift. To test this hypothesis, we conducted three experiments with a sudden extension of the range of the stimuli. In Experiment 1, the presentations proceeded smoothly from one block to the next without an interruption or warning at the point of shift. In Experiment 2, the subjects were asked either to maintain their old scales or to construct new ones. In Experiment 3, the subjects were required to do both things in parallel.

\section{EXPERIMENT 1}

\section{Method}

Subjects. The subjects were 37 undergraduate psychology students at Giessen University who participated in partial fulfillment of course requirements or were paid for their participation. About two thirds were women.

Stimuli and Apparatus. The stimulus set consisted of 16 empty squares whose width varied from 12 to $102 \mathrm{~mm}$, in increments of $6 \mathrm{~mm}$, in an arithmetic series. The squares were presented on a 15in. (38-cm) monochrome computer monitor. The resolution was $640 \times$ 400 pixels, and the refresh rate was $72 \mathrm{kHz}$. The squares appeared as bright lines against a dark background in the center of the screen. The lines were 1 pixel thick. The room was dimly lit. The subjects sat about $60 \mathrm{~cm}$ from the monitor.

Design and Procedure. The subjects were divided into two groups. Group $\mathrm{H}(n=19)$ first judged the eight largest squares, whereas Group L $(n=18)$ first judged the eight smallest. After four blocks of trials with the training series, eight blocks of trials with the total series (Squares 1-16) followed. At Stage 1, each square occurred twice in a block, separated by other squares. At Stage 2, each square appeared once in a block. Therefore, every block consisted of 16 presentations. The order was random every time. The subjects were asked to judge each square according to its size using integers from 1 to 6 . They made their responses by pressing the appropriate number key on the computer keyboard. Response time was measured without the subjects' knowing. The screen was blank for $3 \mathrm{sec}$ after a response had been made. The presentations proceeded smoothly from one block to the next without interruption or warning at the point of shift.

\section{Results}

To track the block-by-blockupward and downward movement of the scales, we calculated the average value of the squares assigned to the middle categories (Category 3 or 4 ) in a block. We took this average value as the neutral point (NP) of the series. In Figure 1, the mean NPs are plotted as a function of trial block for either group. (The stimulus values can be converted into millimeters by adding 1 and then multiplying by 6.) One can see that the two curves quickly approach each other after the shift but do not meet at the end of the experimental series. After eight blocks of trials (i.e., 128 presentations), the curves are still 1.7 stimulus steps apart. This difference is significant $[F(1,35)=$ $36.5, p<.0001]$.

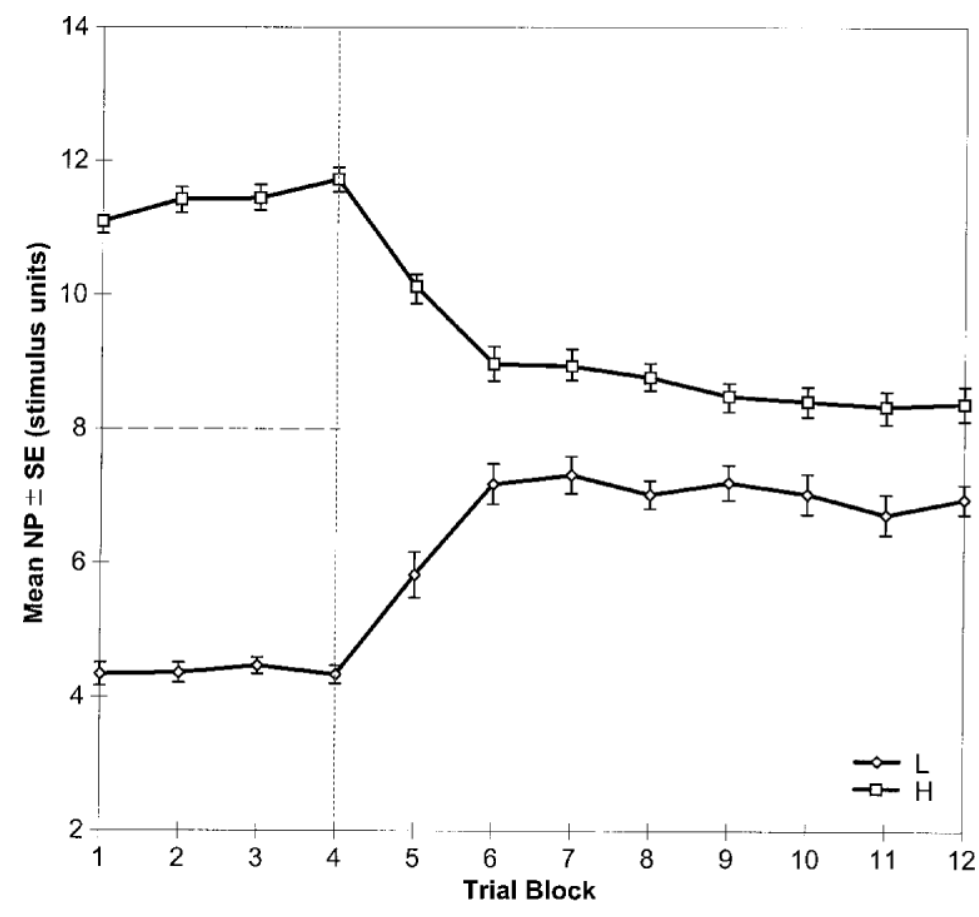

Figure 1. Experiment 1. Upward and downward movements of neutral points (NPs) when no specific instructions were given at the point of shift (dotted vertical line). The dashed horizontal line divides the high $(\mathbf{H})$ training series from the low ( $L$ ) one. Group $L$ judged the low series (Squares 1-8), and Group $H$ judged the high series (Squares 9-16). Vertical bars indicate the standard errors of the means. If adjustment to the total range were complete, the two curves would eventually meet. Physical width of the squares $=6$ (stimulus unit +1$)$. 


\section{Discussion}

The findings are consistent with those reported in the literature. If no specific instructions are given at the point of shift, adjustment to the new range of stimuli will be incomplete. It looks as though the adjustment process stopped after two blocks of trials, since the NP functions fall to an asympotic level after two blocks (32 presentations).

In the next experiment, specific instructions to modify or not to modify scales were given at the point of shift. Because they might have affected not only the NP of the stimulus series but also the general shape of the judgment functions, the magnitude of sequential effects in the judgments, and response time, we analyzed these variables as well.

\section{EXPERIMENT 2}

\section{Method}

Subjects. The subjects were 72 undergraduate students of psychology at Giessen University who participated in partial fulfillment of course requirements or were paid for their participation. About two thirds were women.

Stimuli and Apparatus. The stimuli were identical to those in Experiment 1 . They were presented on a 19-in. $(48-\mathrm{cm})$ color monitor. The resolution was $800 \times 600$ pixels, and the refresh rate was $85 \mathrm{~Hz}$.

Design and Procedure. The design and procedure were the same as before, except that at the point of shift, half the members of Groups $\mathrm{H}$ and $\mathrm{L}$ were asked to keep to their old scales (Subgroups $\mathrm{HO}$ and LO), whereas the other half were asked to construct new ones (Subgroups HN and LN). The label old or new appeared at the bottom of the screen on every trial to remind the subjects of the target scale. Of course, no information was given about the nature of the change, nor of its direction; the subjects were only just asked to judge another series of squares. The wording of the instructions is given in the Appendix. Because past experience had shown us that the scales would reach their final levels after fewer than two blocks of trials, we restricted the number of test blocks to four. As a minor change, we had the subjects judge the squares on a 5-point scale because the NPs could then be calculated directly by averaging the values of the squares assigned to the middle category (Category 3 ).

\section{Results}

Neutral points. Figure 2 shows the block-by-blockupward and downward shifts of the NPs for each subgroup. One can see that Subgroups HO and LO were still about five stimulus steps apart at the end of the experiment, whereas Subgroups HN and LN came very close to each other. For an analysis of the asymptotic portion of the curves, we combined Blocks 3 and 4 and, also, Blocks 7 and 8 , into one larger block. We then computed the NPs for these larger blocks in the same way as before (see Table 1). A comparison between the training and the test levels showed that Subgroups HN and LN shifted their NPs by about three stimulus steps, whereas Subgroups $\mathrm{HO}$ and LO shifted theirs by only $1-1.5$ steps. This difference is significant $\left[F_{\mathrm{HO}}(1,34)=38.7, p<.0001\right.$; and $\left.F_{\mathrm{LO}}(1,34)=7.92, p<.008\right]$, nevertheless. The difference $(M=5.2)$ in the test levels between Subgroups HO and LO is also significant $[F(1,70)=130.7, p<.0001]$,

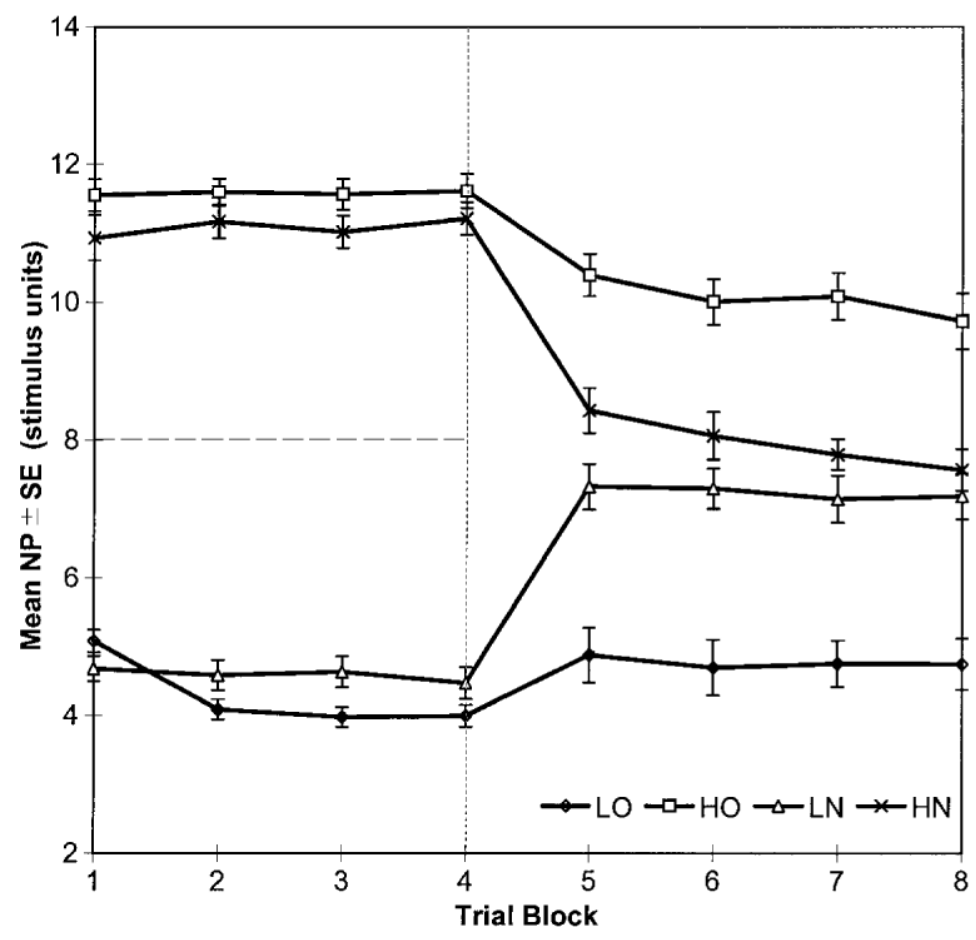

Figure 2. Experiment 2. Upward and downward movements of neutral points (NPs) when instructions to construct new scales $(N)$ or to maintain the old ones $(O)$ were given at the point of transition from the training to the test series (dotted vertical line). The subjects were for the most part able to follow both sets of instructions. 
whereas the difference $(M=0.5)$ between Subgroups HN and LN is not. It should be noted that the power of the experiment to detect a difference of less than 0.5 stimulus steps was only .10.

Judgment functions. Again we combined Blocks 7 and 8 into one larger block. Figure 3 shows the mean judgment functions for the subjects given no instructions in that block. If adjustments were complete, the two curves would be linear. However, they are concave downward for Group L and concave upward for Group H. This must mean that the subjects were able for the most part to maintain their old scales throughout of the experiment.

Sequential effects. Sequential effects must not be confused with pseudosequential effects. Pseudosequential effects can arise when the scales are shifted into their new positions (DeCarlo \& Cross, 1990). To eliminate such effects, we again restricted the analysis to the asymptotic portion of the curves (Blocks 7 and 8). At that point, the scales had reached their final levels. Pseudosequential effects can also arise from the pooling of individual data (Haubensak, 1993). To eliminate individual differences, we employed the pure-error method (DeCarlo \& Cross, 1990; Schifferstein \& Kuiper, 1997). First, we fitted thirdorder polynomials to the judgment functions for each individual separately. In $75 \%$ of the cases, the polynomials accounted for more than $90 \%$ of the judgment variance and in $25 \%$ for more than $95 \%$. We then calculated the "error" residuals by subtracting the estimated mean judg- ment for each stimulus from each individual judgment of that stimulus. From the pooled data $(n=2,232)$, we calculated the correlations between successive residuals for Subgroups HO, LO, HN, and LN. The first-order (i.e., lag 1) autocorrelation coefficients were, in order, .11,.11,.21, and .01. Except in the case of the Subgroup LN, these coefficients were significant; the second-order (i.e., lag 2) coefficients were not.

Response time. Again we skipped the transitional phase, restricting the analysis to the larger blocks of trials made up of Blocks 7 and 8 . The response time (RT) distributions were positively skewed, as usual. We therefore logtransformed the response-time data. In addition, we eliminated all values beyond $\pm 2 z$ from the analysis. This reduced the positive skew to 0.5 . To examine the effects of stimulus age, or familiarity, we grouped the stimuli into old $(\mathrm{O})$ and new $(\mathrm{N})$. The old stimuli were those from the training series, and the new ones were those added after the shift. Furthermore, we called the relationship between the stimuli and target context congruous when an old stimulus was to be rated on the old, subrange-related scale or when a new stimulus had to be rated on the new, totalrange related scale. We called the relationship incongruous when an old stimulus had to be judged on the new scale or a new stimulus had to be judged on the old scale.

In Figure 4, ln RT is shown as a function of stimulus age. The data for old Squares 1-8 and for new Squares 916 came from Group L, whereas those for old Squares 9-

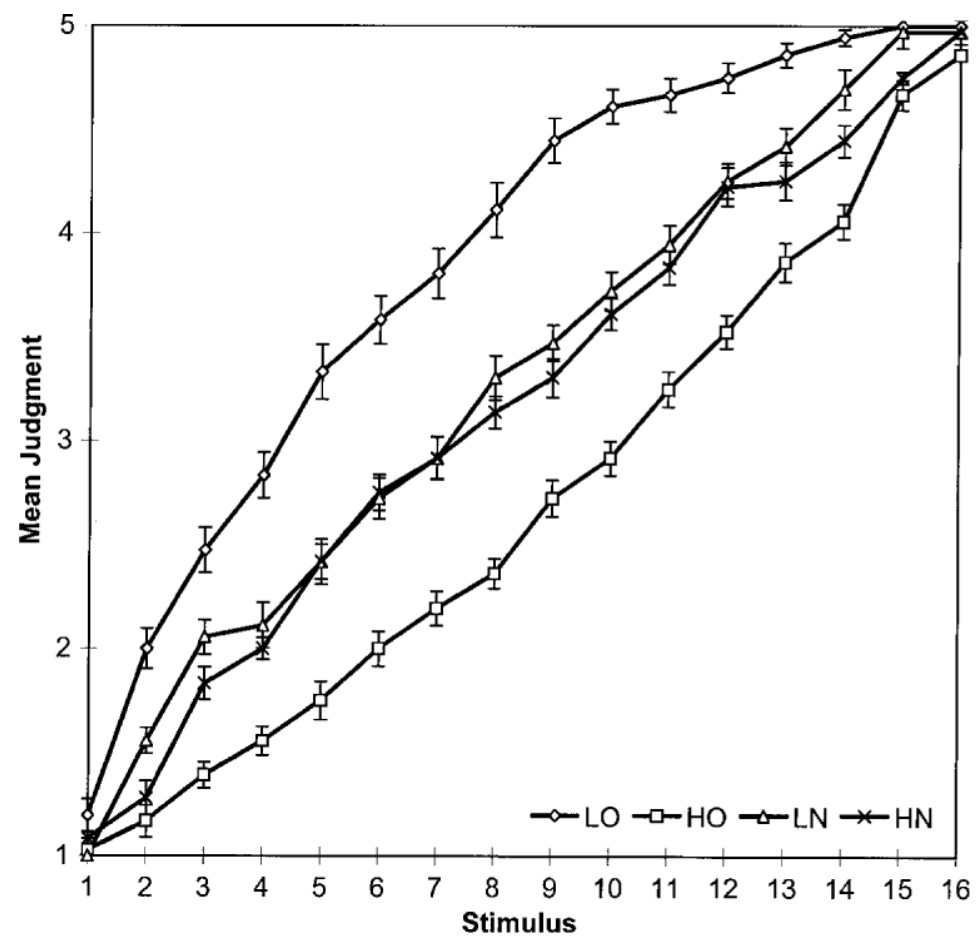

Figure 3. Experiment 2. The last two blocks of test trials (Blocks 7 and 8) combined into one larger block. The mean judgment functions for Subgroups HO, LO, HN, and LN. For an explanation of the abbreviations, see Figures 1 and 2 . 


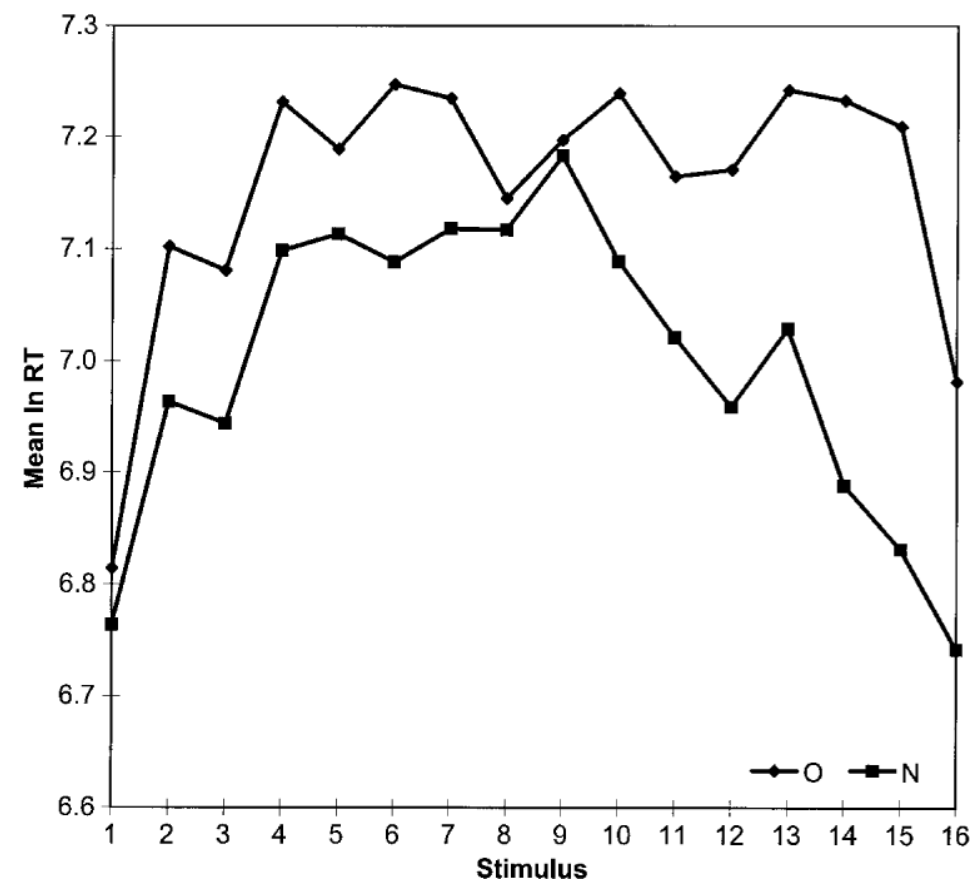

Figure 4. Experiment 2. The last two blocks of test trials (Blocks 7 and 8) combined into one larger block. Mean In RT as a function of stimulus value. The parameter is stimulus age $(\mathrm{O}=$ old, $\mathrm{N}=$ new $)$.

16 and for new Squares 1-8 come from Group H. One can see that the responses to the smallest (Square 1) were the fastest, the responses to the largest (Square 16) were next fastest, and the responses to the medium squares were then slowest. It is interesting that the squares defining the inner endpoints of the old subrange (Squares 8 and 9) were no exception, that is, they did not stand out much against the adjacent ones as far as response time is concerned. The same holds true for the judgment variance (not shown in the figure).

Table 2 shows the geometric means of RT (i.e., mean $\ln$ RT retransformed to the numerus) for the groups of old and new stimuli and, also, for the groups of congruous and incongruous stimuli. One can see that the responses to the old stimuli were $193 \mathrm{msec}$ slower on the average than were the responses to the new ones. The responses to incongruous stimuli were $135 \mathrm{msec}$ faster than were the responses to the congruous ones. A 2 (stimulus age) $\times 2$ (congruity) Latin squares repeated measures analysis (Winer, 1962, Plan 5, pp. 539-543) indicated that the effect of stimulus age was significant $[F(1,140)=24.1, p<.0001]$. The effect of congruity was also significant $[F(1,140)=$ $11.9, p<.001]$, whereas the interaction between the two factors was not.

\section{Discussion}

The results are consistent with the hypothesis that instructions to modify or not to modify scales affect the weighting of successive stimulus contexts. Individuals are for the most part able to construct new scales if instructed to do so, and they are able to maintain their old scales as well. Consequently, previous experience in a series of stimuli does not always have profound, or dramatic, effects on the judgment of a second. It does have an effect when subjects are made to guess about the nature of the experiment.

Frame of reference. Because the first-order sequential effects were so small, one must conclude that the subjects made their judgments relative to a fixed frame of reference rather than to the immediately preceding stimulusresponse pair. This pair might be forgotten in a few seconds (cf. DeCarlo \& Cross, 1990). It is surprising that the responses to the stimuli formerly defining the inner endpoints in the training series (Stimulus 8 or 9) were among the slowest (see Figure 4). The variance of the responses to these stimuli was also large. Hence, it is unlikely that they were the only ones that acted as points of reference, or standards, for the judgment to the other stimuli. On the other hand, it is not necessary to assume that every stimulusresponse is stored in long-term memory for later use as a standard. Rather, we assume that response habits developed as the presentations proceeded. These habits might generalize to a wide range of stimuli. Thus, a square might look small even though subjects are unable to identify it individually. The consistency model of Haubensak (1992) is an elaboration of this idea. Ward (1987) might have had a similar idea in mind when he suggested that memory for past context takes the form of a sort of "fuzzy mapping" 


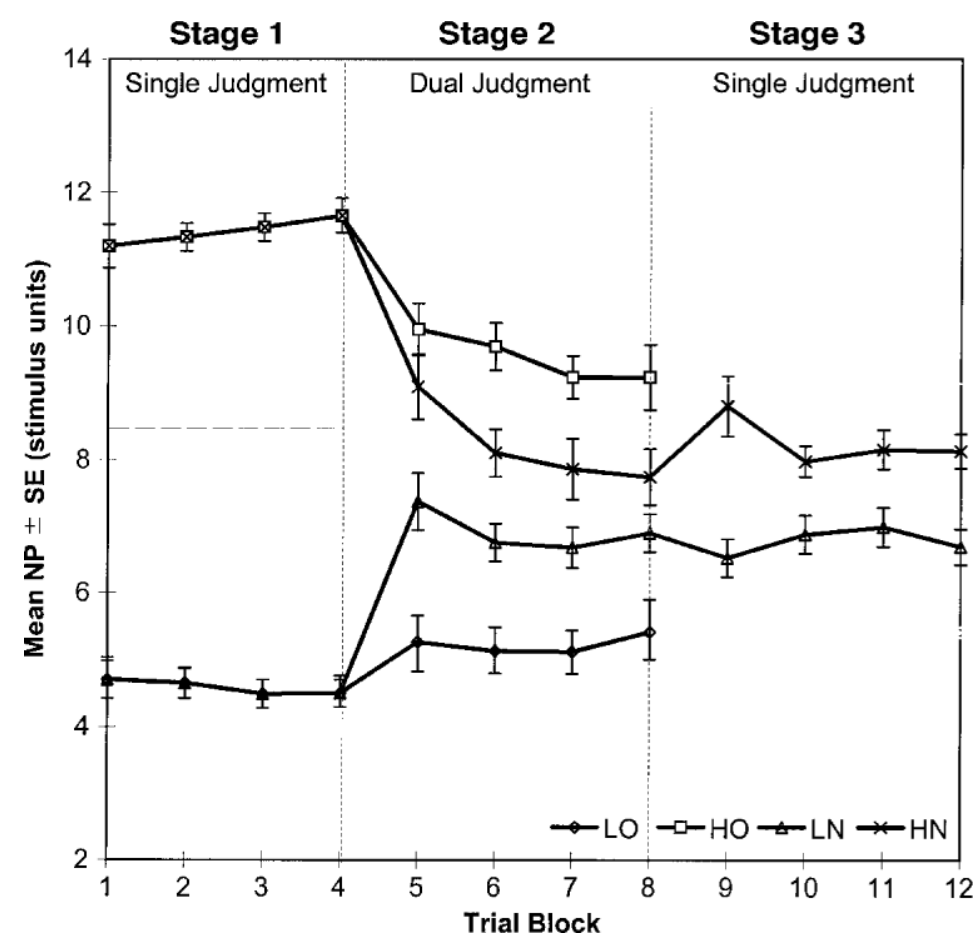

Figure 5. Experiment 3. Upward and downward movements of neutral points (NPs). Stage 1, single judgment: training (H or L). Each stimulus had to be judged relative to the training series. Stage 2 , dual judgment: test stage (Squares 1-16). The same subjects judged the same stimulus twice, relative to the training series $(O)$ and to the test series $(N)$. Therefore, the curves are split here. Stage 3, single judgment: Subjects judged each stimulus relative to the current series only (Squares 1-16).

from stimuli to responses. Theories such as these do not ascribe a special status to the extreme stimuli, except that these are discriminated best because they have adjacent stimuli on only one side.

Response interference hy pothesis. Although coming close, the subjects were unable to conform to the instructions completely. Group LO, for example, assigned the largest square of the training series (Square 8) to Category 4 rather than to Category 5 after the shift. There are two possible reasons for this partial failure. Instruction $\mathrm{O}$ required the subjects to assign the new stimuli to the highest or lowest category. This would make the judgment distributions heavily skewed. Because subjects want to assign the same number of stimuli to each category (the frequency tendency; see Parducci, 1963; Stevens \& Galanter, 1957), they might have shifted their scales only slightly. The scales start shifting before the skewing of the stimulus frequencies has become fully apparent, however (Haubensak, 1992; Parducci \& Wedell, 1986). Moreover, keeping a written record of one's own responses has surprisingly little effect on the strength of the frequency tendency, though it makes the skewing immediately apparent (Parducci, 1963). Therefore, we give less credit to this interpretation. Rather, we assume that response habits become conditioned to the stimuli as the presentations proceed. After an extension of the stimulus range, the previously acquired response habits might bias responses to the new context.
The new responses in turn might bias the old ones when subjects are required to judge the stimuli relative to the past series. This backward interference could be even stronger than forward interference because new responses are constantly reinforced by the new context, whereas old response habits are not.

One benefit of the response interference hypothesis is that it accounts for the effect of stimulus age on reaction time. Because associative interference retards responses (Anderson, 1995), responses to old stimuli should be slower than responses to new ones, which have no past. The present results are consistent with this hypothesis. Responses to incongruous stimuli might be faster because only a few response alternatives are left for them. A new stimulus, for example, falls into to the highest or lowest category when it has to be rated on the old, subrange-related scale. Similarly, an old stimulus must be rated either higher or lower than before when it has to be rated on the new, total range-related scale.

Multiple contexts. The results of Experiment 2 show that subjects are able to maintain their old scales or, alternatively, to construct new ones if instructed to do so. The next question is whether people can do both things in parallel. Evidence for this assumption comes from everyday experience; for example, statements like the following are readily accepted: "For a briefcase, this weight is heavy but for a suitcase, it is light." Obviously, people have little dif- 
ficulty in dealing with two different contexts for the same stimulus attribute. According to unlearning, or adaptation, theories of judgment (e.g., Helson, 1964; Johnson, 1949), the establishment of two contexts for the same stimulus attribute should be impossible, because the current context would eliminate the previous one as the presentations proceed. Helson as well as Johnson have proposed mathematical formulations of this idea. In memory psychology, unlearning or adaptation theories have their counterpart in assimilation, or substitution, theories (e.g., Loftus \& Loftus, 1980). We would rather assume that multiple contexts for the same attribute (e.g., size) can exist in memory. Experiment 3 was designed to test this hypothesis by asking the subjects to judge the same stimulus twice in succession, first in the past context and then in the current one, or vice versa. This might be called the dual-judgment procedure. The expectation was that the subjects would be able to maintain their old scales while constructing new ones. An additional expectation was that by contrasting the two scales, more separation between the old scales would be produced, because the subjects might overcompensate for a possible confusion of old and new responses (cf. Wegener, 1997). For a control, we added a third stage to the experiment. Here, the subjects had to make only one judgment to each stimulus relative to the shifted context.

\section{EXPERIMENT 3}

\section{Method}

The subjects were 42 undergraduate psychology students who participated in partial fulfillment of course requirements. The stimulus material and procedures were the same as in Experiment 2, except that at Stage 2, each stimulus was repeated immediately. Again the screen was blank for $3 \mathrm{sec}$ between repetitions. At the point of transition, from training to the test series, the subjects of both Groups $\mathrm{H}$ and $\mathrm{L}$ were told that each square would be presented twice in succession. Half the subjects were asked to judge the square relative to the old series (the training series) on the first presentation and relative to the current series (the test series) on the second (Subgroups LON and HON). For Subgroups LNO and HNO, the order of judgments was reversed. As a reminder, the label old or new appeared at the bottom of the screen on each trial. At Stage 3, each stimulus was to be judged in relation to the current series only. Each stage consisted of four blocks of trials. Again, no information was given about the nature of the shift; the subjects were merely asked to judge another series of squares. The wording of the instructions is given in the Appendix.

\section{Results}

Two subjects who made inconsistent judgments were eliminated from the analysis under the assumption that they had misunderstood the instructions. Because neither the main effect of judgment order nor the interaction with group was significant, we pooled the data from the two different judgment orders.

Neutral points. Figure 5 shows the block-by-blockupward and downward movements of the NPs. It should be remembered that there were only two groups of subjects, $\mathrm{H}$ and $\mathrm{L}$, and that the instructions had been manipulated as a within-subjects variable; that is, the same subjects judged the same stimulus twice, relative to the training se- ries $(\mathrm{O})$ and relative to the test series $(\mathrm{N})$. Nevertheless, the curves are similar to those presented in Figure 2. The final levels (Blocks 7 and 8 combined into one larger block) are given in Table 1. One can see that Groups $\mathrm{H}$ and L differ by 4.2 stimulus steps when the target context was the training series, whereas they differ by only 1.2 stimulus steps when the target context was the test series. A training $\times$ target context analysis of variance (ANOVA) with repeated measures on context indicates that the between-groups effect of the training series is significant $[F(1,38)=47.8, p<.0001]$. The effect is significant at both levels of the target-context factor $\left[F_{\mathrm{O}}(1,38)=89.0\right.$, $p<.0001$; and $\left.F_{\mathrm{N}}(1,38)=5.7, p=.02\right]$. Furthermore, the effect of the target context is significant within both training groups $\left[F_{\mathrm{H}}(1,38)=12.4, p=.001\right.$; and $F_{\mathrm{L}}(1,38)=$ $15.7, p<.001]$.

Other measures. Because the judgment functions are nearly identical to those in Figure 3, they are not shown again. To test for sequential effects, we proceeded as before; that is, we fitted, for each subject separately, third-order polynomials to the new and, then, to the old judgments across Trial Blocks 7 and 8 . We then computed the "error" residuals by subtracting the estimated mean judgments for each stimulus from the individual judgments of that stimulus. The correlations between successive old/new residuals and between successive new/old residuals were $r(672)=$ $.12, p=.001$, and $r(608)=.20, p<.001$, respectively.

Finally, we analyzed response time in the same manner as before. Only the first judgment of a stimulus was entered into the analysis, because the subjects might have started making their second judgment during their first one. Again, the stimuli were grouped into old and new, congruous and incongruous. The geometric means of RT for these groups are presented in Table 2. A 2 (stimulus age) $\times 2$ (congruity with target context) Latin squares repeated measures analysis (see Winer, 1962, pp. 539-543, Plan 5) revealed that the effect of stimulus age was marginally significant $[F(1,76)=3.7, p=.06]$. All other effects, including the interaction between stimulus age and congruity, were not.

Stage 3. From Figure 5 one can see that under Instruction N, the NPs first moved toward those found under Instruction $\mathrm{O}$ and then returned to their former level. A 2 (group) $\times 2$ (stage) repeated-measures analysis indicated that there was no significant difference in the final levels between Stages 2 and 3. An analysis of the response time data shows that mean RT was about 300 msec slower on the first half of Stage 3 (Blocks 9 and 10 combined) than on the second half (Blocks 11 and 12 combined).

\section{DISCUSSION}

The results of Experiment 3 show that people can maintain their old scales while constructing new ones. The results are consistent with the hypothesis that two contexts can coexist in memory for the same stimulus attribute (e.g., size). Contrary to expectation, the dual-judgment procedure did not produce contrast effects (e.g., by prompting the subjects to rate a square smaller than perceived in 
the first judgment and larger in the second). Rather, the dual-judgment procedure produced assimilation. This is indicated by the larger separation between curves LN and $\mathrm{HN}$ in Figure 5 as compared with those in Figure 2 (the single-judgment task).

It is unlikely that this assimilation was caused by a tendency of the subjects to repeat the first judgment on the immediately following presentation of the same stimulus, since the correlations between successive error residuals were not much different from those in the single-judgment task (Experiments 1 and 2). Rather, we assume that the dual-judgment task produced a slightly greater amount of interference than did the single-judgment task because the subjects had to keep both the old and the new contexts in mind, whereas in the single-judgment task, they could concentrate on one and ignore the other. In short, one's making two judgments about the same stimulus might be more demanding than making just one. Consistent with this interpretation, mean RT was $128 \mathrm{msec}$ longer in the dualjudgment than in the single-judgment task.

Because the old response habits were revived in Stage 2 over and over again, there might also have been more interference at the start of Stage 3 than later on. As a result, the new NPs approached the old ones on the first block of trials and then returned to their former level. Consistent with this interpretation, the responses to the stimuli were much slower in the first two blocks of trials than in the last two (see Table 2).

\section{CONCLUSIONS}

Psychophysical scales are extremely malleable. Subjects can, for example, be trained to use experimenter-defined judgment functions (Baird, Kreindler, \& Jones,

Table 1

Final Levels of the Neutral Points

\begin{tabular}{|c|c|c|c|c|}
\hline Group & Instruction & $n$ & $M$ & $S D$ \\
\hline \multicolumn{5}{|c|}{ Experiment 1} \\
\hline $\mathrm{L}$ & & 18 & 7.2 & 0.9 \\
\hline $\mathrm{H}$ & & 19 & 8.9 & 0.8 \\
\hline \multicolumn{5}{|c|}{ Experiment 2} \\
\hline $\mathrm{L}$ & $\mathrm{O}$ & 18 & 4.7 & 1.4 \\
\hline $\mathrm{L}$ & $\mathrm{N}$ & 18 & 7.2 & 1.4 \\
\hline $\mathrm{H}$ & $\mathrm{O}$ & 18 & 9.9 & 1.5 \\
\hline $\mathrm{H}$ & $\mathrm{N}$ & 18 & 7.7 & 1.1 \\
\hline \multicolumn{5}{|c|}{ Experiment 3 , Phase 2} \\
\hline \multirow[t]{3}{*}{$\mathrm{L}$} & & 20 & & \\
\hline & $\mathrm{O}$ & & 5.2 & 1.4 \\
\hline & $\mathrm{N}$ & & 6.7 & 1.3 \\
\hline \multirow[t]{3}{*}{$\mathrm{H}$} & & 20 & & \\
\hline & $\mathrm{O}$ & & 9.4 & 1.5 \\
\hline & $\mathrm{N}$ & & 7.9 & 1.8 \\
\hline \multicolumn{5}{|c|}{ Experiment 3 , Phase 3} \\
\hline $\mathrm{L}$ & $\mathrm{N}$ & 20 & 6.9 & 1.2 \\
\hline $\mathrm{H}$ & $\mathrm{N}$ & 20 & 8.2 & 1.1 \\
\hline
\end{tabular}

Note-The neutral points are given in stimulus units. Physical width = 6 (stimulus unit +1 ).
Table 2

Geometric Means of the Final Levels of Reaction Time (in Milliseconds) for Blocks 7 and 8 Combined

\begin{tabular}{cccc}
\hline \multirow{2}{*}{ Experiment } & Condition & \multicolumn{2}{c}{ Stimulus Age } \\
\cline { 3 - 4 } & & Old & New \\
\hline 1 & & 1,114 & 1,024 \\
2 & Congruous stimuli & 1,289 & 1,096 \\
& Incongruous stimuli & 1,359 & 1,164 \\
3 & & 2,184 & 1,032 \\
& Congruous stimuli & 2,194 & 1,860 \\
& Incongruous stimuli & 2,173 & 1,948 \\
& &
\end{tabular}

Note-For Experiment 3, reaction times are for the dual-judgment procedure and only for first judgments.

1971; West, Ward, \& Kosla, 2000). Our study is another case in point, showing that the weighting of successive contexts is governed by instructions. The importance of the instruction variable has also been emphasized by Parducci, Knobel, and Thomas (1976), who asked their subjects to rate an intermingled series of smaller squares and larger circles with a single set of categories. One group of subjects was asked to establish separate scales for each domain (squares and circles), whereas controls were asked to ignore the difference in shape-that is, to combine the squares and circles into a single context. Most of them were able to do so, although a larger gap in size between the two sets of figures encouraged separation rather than integration.

The experiments of Parducci et al. (1976), however, differ from ours in some respects. First, the contexts were made up of different types of figures (circles and squares), whereas in our experiments the contexts were successive stages of the experiment. The findings suggest that previous presentations are as effective as a context cue as are such sensory attributes as stimulus shape or stimulus color. Second, we manipulated the instructions not only as a between-subjects variable (as Parducci et al. did) but also as a within-subjects variable. The findings suggest that individuals are able to establish two multiple scales for the same attribute (e.g., size) and that they are able to use them rather independently. The results may be interesting for scaling theory and scaling practice as well.

There are limits to the independence of successive contexts, nevertheless. The subjects were not able to keep their old scales completely unchanged, nor were they able construct new scales independently of the old ones. We assume that response habits develop as the presentations proceed. These habits can bias responses to the new context. Similarly, implicit responses to the new context might bias the old ones when subjects are required to judge the stimuli relative to the past series. The claim that conditioning processes contribute to the formation of scales is not new at all. It has been made by many authors (e.g., Guilford, 1954; Haubensak, 1992; Siegel \& Siegel, 1972; Tresselt, 1947; Tresselt \& Volkmann, 1942; Wedell, 1984). Tresselt and Volkman, for example, proposed the hypothesis that the principles of judgment are the principles of conditioning (cf. Guilford, 1954). Our findings lend sup- 
port to this hypothesis. The findings also indicate that the establishment of contexts is primarily governed by instructions under otherwise similar conditions.

\section{REFERENCES}

ANDERson, J. R. (1995). Learning and memory: An integrated approach. New York: Wiley.

Baird, J. C., Kreindler, M., \& Jones, K. (1971). Generation of multiple ratio scales with a fixed stimulus attribute. Perception \& Psychophysics, 9, 399-403.

DeCarlo, L. T., \& Cross, D. V. (1990). Sequential effects in magnitude scaling: Models and theory. Journal of Experimental Psychology: General, 119, 375-396.

Di Lollo, V. (1964). Contrast effects in the judgment of lifted weights. Journal of Experimental Psychology, 68, 383-387.

Di Lollo, V., \& CAsseday, J. H. (1965). Graded contrast effects in the judgment of lifted weights. Journal of Experimental Psychology, 70, 234-235.

Guilford, J. P. (1954). Psychometric methods (2nd ed.). New York: McGraw-Hill.

Haubensak, G. (1992). The consistency model: A process model for absolute judgments. Journal of Experimental Psychology: Human Perception \& Performance, 18, 303-309.

HaubensaK, G. (1993). Sequenzeffekte in absoluten Urteilen? Kritisches zur Methode. [Sequential effects in absolute judgments? Critical comments on the method.] Zeitschrift für Experimentelle und Angewandte Psychologie, 39, 101-113.

Helson, H. (1964). Adaptation-level theory: An experimental and systematic approach to behavior. New York: Harper \& Row.

Johnson, D. M. (1949). Learning function for a change in the scale of judgment. Journal of Experimental Psychology, 39, 851-860.

Loftus, E. F., \& Loftus, G. R. (1980). On the permanence of stored information in the human brain. American Psychologist, 35, 409-420.

PARDUCCI, A. (1954). Learning variables in the judgment of single stimuli. Journal of Experimental Psychology, 48, 24-30.

Parducci, A. (1963). Range-frequency compromise in judgment. Psychological Monographs, 77 (2, Whole No. 565), 1-29.
Parducci, A., Knobel, S., \& Thomas, C. (1976). Independent contexts for category ratings: A range-frequency analysis. Perception \& Psychophysics, 20, 360-366.

Parducci, A., \& Wedell, D. H. (1986). The category effect with rating scales: Number of categories, number of stimuli, and method of presentation. Journal of Experimental Psychology: Human Perception \& Performance, 12, 496-516.

Schifferstein, H. N. J., \& Kuiper, W. E. (1997). Sequence effects in hedonic judgments of taste stimuli. Perception \& Psychophysics, 59, 900-912

Siegel, J. A., \& Siegel, W. (1972). Absolute judgment and pairedassociate learning: Kissing cousins or identical twins? Psychological Review, 70, 300-316.

Stevens, S. S., \& Galanter, E. H. (1957). Ratio scales and category scales for a dozen perceptual continua. Journal of Experimental Psychology, 54, 377-411.

Tresselt, M. E. (1947). The influence of amount of practice upon the formation of a scale of judgment. Journal of Experimental Psychology, 37, 251-260.

Tresselt, M. E., \& Volkmann, J. (1942). The production of uniform opinion by non-social stimulation. Journal of Abnormal \& Social Psychology, 37, 234-243.

WARD, L. M. (1987). Remembrance of sounds past: Memory and psychophysical scaling. Journal of Experimental Psychology: Human Perception \& Performance, 13, 216-227.

Ward, L. M., \& LockHeAD, G. R. (1970). Sequential effects and memory in category judgments. Journal of Experimental Psychology, 84, 27-34.

WEDELL, D. H. (1984). A process model for psychophysical judgment. Dissertation Abstracts International, 45, 3102-B (University Microfilms 8428589).

Wegener,D. T. (1997). The flexible correction model: The role of naive theories of bias in bias correction. Advances in Experimental Social Psychology, 29, 141-208.

West, R. L., Ward, L. M., \& Kosla, R. (2000). Constrained scaling: The effect of learned psychophysical scales on idiosyncratic response bias. Perception \& Psychophysics, 62, 137-151.

WINER, B. J. (1962). Statistical principles in experimental design. New York: McGraw-Hill.

\section{APPENDIX}

\section{Specific Instructions in Experiment 2 (English Translation)}

Group $\mathbf{O}$. We now continue to the second stage of the experiment. Again, you will be shown squares. We want to know whether you are able to retain the scale that was established during the first stage. The more successful you are in preserving the old scale, the better. If you had called a square 1 during the first stage of the experiment, press Key 1, if you had called it 2, press Key 2, and so on. Use integers 1-5 again. To remind you of the reference, the label old appears at the bottom of the screen on every presentation.

Group N. Now we come to the second stage of the experiment. Again, you will be shown squares. We want to know whether you are able to establish a new scale. The more successful you are in ignoring the scale that was established during the first stage, the better. If you think that Category 1 is appropriate now, press Key 1, if you think that Category 2 is appropriate, press Key 2, and so on. Use integers 1 to 5 again. To remind you of the reference, the label new appears at the bottom of the screen on every presentation.

\section{Specific Instructions in Experiment 3, Stage 2}

Now we come to the second stage of the experiment. Again you will be shown squares. We would like to know whether you are able to judge the sizes of these squares in different contexts. Each square appears twice in succession. If the label old appears at the bottom of the screen, judge the square relative to the series presented at the first stage of the experiment. If the label new appears, judge the same square relative to the current series. Use integers 1-5 again. The more successful you are in isolating the old or new context by your judgments, the better. Be as careful as before. Respond to every square even if the decision seems difficult for the first squares.

\section{Specific Instructions in Experiment 3, Stage 3}

Now we come to the third and last stage of the experiment. Again you will be shown squares. Each square appears only once in a block. Please judge the square relative to the current series. Use integers 1-5 again. The more successful you are in establishing a new scale, the better. 